\title{
DEPRESSION AND ITS ASSOCIATED FACTORS: A STUDY ON CAREGIVERS OF PSYCHIATRIC PATIENTS
}

\author{
Nomita Imtiaz, Shiraz Hussain, Siddique Kakar*, Sana Khan, Mohsin Alimudin**, Mohammad Kamran***, Sohail Ali \\ Armed Forces Institute of Mental Health/National University of Medical Sciences (NUMS) Rawalpindi Pakistan, *Pakistan Naval Ship Shifa Hospital Karachi \\ Pakistan, ${ }^{* *}$ Combined Military Hospital Panoaqil/National University of Medical Sciences (NUMS) Pakistan, ${ }^{* * *}$ Combined Military Hospital Malir/National \\ University of Medical Sciences (NUMS) Pakistan
}

\section{ABSTRACT}

Objective: To determine the frequency and association of depression among caregivers of psychiatric patients. Study Design: Cross-sectional study.

Study place and duration: Outpatient Department of a Tertiary Care Hospital, Rawalpindi, from Sep 2016 to Apr 2017. Methodology: A total of 200 caregivers of psychiatric patients were included in this study. Each caregiver was interviewed by a consultant psychiatrist to measure the frequency of depression according to International Classification of Disease-10 (ICD10) criteria and Hospital Anxiety and Depression Scale (HADS) score of $>8$. Socio-demographic factors were associated with presence of depression by using chi-square test.

Results: The mean age observed was $35.36 \pm 8.39$ years. There were $84(42 \%)$ males and $116(58 \%)$ females. Depression was found in $95(47.5 \%)$ caregivers of psychiatric patients in our analysis. Female gender, low socio-economic status and type of psychiatric illness had significant relationship with presence of depression among our target population.

Conclusion: Depression was a highly common undiagnosed entity among the caregivers of psychiatric patients. Female caregivers or caregivers with low socio-economic status should be screened especially for depression in addition to caregivers of patients suffering from psychotic illness.

Keywords: Caregivers, Depression, Psychiatric disorders.

How to Cite This Article: Imtaiz N, Hussain S, Karar S, Khan S, Alimudin M, Kamran M, Ali S. Depression and Its Associated Factors: A Study on Caregivers of Psychiatric Patients. Pak Armed Forces Med J 2021; 71(6): 1933-1936. D Doi: https://doi.org/10.51253/pafmj.v6i6.2971

This is an Open Access article distributed under the terms of the Creative Commons Attribution License (https://creativecommons.org/licenses/by-nc/4.0/), which permits unrestricted use, distribution, and reproduction in any medium, provided the original work is properly cited.

\section{INTRODUCTION}

Over the past several decades, as medical science has achieved milestones in almost every quarter but global burden of diseases yet paralleled. ${ }^{1}$ Today, medical professionals are cognizant of fact that depression is the fourth leading cause of global disease burden. In developing countries like Pakistan, up to $20 \%$ of cases who attend primary health care, have been found suffering from linked anxiety and depression but the symptoms of these conditions remain unrecognized. ${ }^{2}$

Caregiving has been an important subject especially regarding the psychiatric patients. All over the world, the subject of caregiving has gained importance as it has vital role in achieving optimal remission in psychiatric illness of the patient. Caregiving is a complex phenomenon with many dimensions. Approximately $1.5-2.5 \%$ of total national healthcare budgets based on estimations in United Kingdom, Netherlands, France and United States have been utilized in caregiving of the patients suffering from schizophrenia. ${ }^{2}$ In addition to economic burden there is a lot of emotional

Correspondence: Dr Nomita Imtiaz, Consultant Psychiatrist, Armed Forces Institute of Mental Health, Rawalpindi Pakistan

Received: 14 Jun 2019; revision received: 17 Aug 2019; accepted: 19 Aug 2019 burden as well. Emotional trauma of having a relative suffering from chronic illness and slow recovery may pose caregiver towards additional stress. Absenteeism from work and lack of emotional support also prone the caregiver towards various psychological problems which can cause poor quality of life for both the patient and caregiver. ${ }^{3}$

Care giving neither causes depression, nor every caregiver go through same emotional turmoil. Sometimes caregivers become over enthusiastic and in enthusiasm of providing the best care to the patient, they forget their own needs and ignore themselves. Time barred negative feelings of caregivers, over a period are often compounded by social and emotional stresses leading to symptomatic depression thus warning signs of depression. Concerns about depression arise when these signs persist for a certain timeframe thus impacting personal life of caregiver. ${ }^{3}$

Almost one-third of all caregivers are balanced between employment and care giving responsibilities. ${ }^{4}$ Out of this proportion, two-thirds report conflicts in roles that require them to rearrange their work schedules, disturbing routine working and resort to leaves of absence. Term of care giving burden was coined to 
sum up all the feelings and needs which arise in the caregiver during the process of caregiving. This holds true for all the illnesses but especially for the chronic illnesses including the mental health problems. Early detection and addressing this burden with direct questioning or using specific tools may help both the patient as well as the caregiver.

A study done by Rodrigo et al, showed that among 80 caregivers of patients with psychiatric illness, $37.5 \%$ were found to be positive for depression. ${ }^{4}$ As such depression is likely to be high among caregivers. The result of another study conducted by Imran et al, showed that $85 \%$ of caregivers of psychiatric patients were depressed. ${ }^{5}$ Whereas in a study done by Alvi et al, it was revealed that depression and anxiety were present in $>50 \%$ of the caregivers of psychiatric patients. 6

Only two studies conducted in Pakistan on this subject have depicted significantly variable results. We need to generate our own data specific to our part of the world in order to understand this phenomenon in context of our own culture. Every culture and religion has its own view regarding caregiving phenomenon which can affect the process of care giving in number of ways. Therefore, this study was planned with the objective to determine the prevalence and correlates of depression among caregivers of psychiatric patients.

\section{METHODOLOGY}

This cross-sectional study was conducted at outpatient department of a Tertiary Care Hospital, Rawalpindi from September 2016 to April 2017. Sample size of 200 cases was calculated with $95 \%$ confidence level, $5 \%$ margin of error and taking expected prevalence of depression i.e., 4 (37.5\%) among caregivers of psychiatric patients was calculated by WHO sample size calculator. Non-probability consecutive sampling technique was used. Caregivers of psychiatric patients of both genders with age 20-60 years were included in the study.

Inclusion Criteria: Immediate family members (parents, spouse, siblings, daughter/son) of the patient living with the patient, in the same environment, for at least 6 months and involved directly in giving care to the patient for six months or more.

Exclusion Criteria: Caregivers with illicit substance use or past history of psychiatric history were excluded from the study.

Depression was characterized by either a dysphoric mood or loss of interest or pleasure in daily life activities. The mood disturbance must be prominent, relatively persistent and in most of the cases, for at least two weeks. It was measured by Hospital Anxiety and Depression Scale (HADS), 7 of the items in even serial i.e., 2, 4, 6, 8, 10, 12 and 14 relate to depression. Each item on the scale means that a person can score from ' 0 ' and ' 21 '. A cutoff score 8/21 identifies depression. Psychiatric patients were diagnosed patients of schizophrenia, bipolar affective disorder, depression and substance abuse. After taking informed written consent 200 primary caregivers associated with the patients coming in OPD were included in the study. Each caregiver was interviewed and selected for the study by consultant psychiatrist and interviewed in person alone and name was kept confidential. Urdu version of HADS was applied for screening of depression. All the data was collected in a predesigned proforma.

Data analysis was performed by using Statistical Package for the social sciences (SPSS) version 23. Quantitative variables such as age of caregiver and patient as well as the duration of psychiatric illness were presented as Mean \pm SD. Qualitative variables such as gender, depression, type of psychiatric illness, socioeconomic status, and education level was presented as frequency and percentages. Chi square test was applied. The $p$-value $\leq 0.05$ was considered significant.

\section{RESULTS}

A total of 200 caregivers of psychiatric patients were included in this study. Most of the caregivers were between 31-40 years of age. The mean age observed was $35.36 \pm 8.39$ years and mean duration of disease was $7.06 \pm 2.91$ years. There were $84(42 \%)$ males and $116(58 \%)$ females. Most of the caregivers were low educated (Matriculate or intermediate) and had low socioeconomic status. Regarding type of psychiatric illness, 99 (49.5\%) had schizophrenia, 90 (45\%) had bipolar disorders and 11 (5.5\%) had mental and behavioral disorders due to substance abuse.

Frequency of depression among caregivers of psychiatric patients was found in 95 (47.5\%). Depression among caregivers of psychiatric patients was not significant among different age groups $(p=0.461)$ while it was observed that rate of depression was significantly high in females as compared to male caregivers $p=0.001$, similarly depression among caregivers of psychiatric patients was not significant with level of education $p=0.203$ (Table-I). Rate of depression was significant with socio economic status $p=0.024$ and type of psychiatric illness $p=0.023$ while insignificant with duration of disease $p=0.263$ as shown in Table-II. 
Table-I: Association of age, gender and education with the presence of depression.

\begin{tabular}{|c|c|c|c|}
\hline \multirow{2}{*}{ Parameters } & \multicolumn{2}{|c|}{ Depression, $\mathbf{n}(\%)$} & \multirow{2}{*}{$\begin{array}{c}p \text { - } \\
\text { value }\end{array}$} \\
\hline & Yes, $(n=95)$ & No, $(n=105)$ & \\
\hline \multicolumn{4}{|l|}{ Age (Years) } \\
\hline$\leq 30$ & $28(42.4 \%)$ & $38(57.6 \%)$ & \multirow{3}{*}{0.461} \\
\hline $31-40$ & $46(52.3 \%)$ & $42(47.7 \%)$ & \\
\hline$>40$ & $21(45.7 \%)$ & $25(54.3 \%)$ & \\
\hline \multicolumn{4}{|l|}{ Gender } \\
\hline Male & $22(26.2 \%)$ & $62(73.8 \%)$ & \multirow{2}{*}{0.001} \\
\hline Female & $73(62.9 \%)$ & $43(37.1 \%)$ & \\
\hline \multicolumn{4}{|l|}{ Education } \\
\hline Illiterate & $33(57.9 \%)$ & $24(42.1 \%)$ & \multirow{4}{*}{0.203} \\
\hline Metric & $28(39.4 \%)$ & $43(60.5 \%)$ & \\
\hline Intermediate & $27(45.8 \%)$ & $32(54.2 \%)$ & \\
\hline Graduate/Master & $7(53.8 \%)$ & $6(46.2 \%)$ & \\
\hline \multicolumn{4}{|c|}{$\begin{array}{l}\text { Table-II: Association of depression in caregivers of } \\
\text { psychiatric patients with socioeconomic status, type and } \\
\text { duration of illness. }\end{array}$} \\
\hline \multirow{2}{*}{ Parameters } & \multicolumn{2}{|c|}{ Depression, $\mathrm{n}(\%)$} & \multirow{2}{*}{$\begin{array}{c}p \text { - } \\
\text { value }\end{array}$} \\
\hline & Yes, $(n=95)$ & No, $(n=105)$ & \\
\hline \multicolumn{4}{|c|}{ Socioeconomic Status } \\
\hline Low & $59(51.3 \%)$ & $56(48.7 \%)$ & \multirow{3}{*}{0.024} \\
\hline Middle & $29(51.8 \%)$ & $27(48.2 \%)$ & \\
\hline High & $7(24.1 \%)$ & $22(75.9 \%)$ & \\
\hline \multicolumn{4}{|c|}{ Type of Psychiatric illness } \\
\hline $\begin{array}{l}\text { Bipolar affective } \\
\text { disorder }\end{array}$ & $36(40 \%)$ & $54(60 \%)$ & \multirow[b]{2}{*}{0.023} \\
\hline $\begin{array}{l}\text { Mental \& } \\
\text { Behavioural } \\
\text { Disorder Due to } \\
\text { substance abuse }\end{array}$ & $9(81.8 \%)$ & $2(18.2 \%)$ & \\
\hline Schizophrenia & $50(50.5 \%)$ & $49(49.5 \%)$ & \\
\hline \multicolumn{4}{|l|}{ Duration of Illness } \\
\hline 1-6 Years & $36(42.9 \%)$ & $48(57.1 \%)$ & \multirow{2}{*}{0.263} \\
\hline 7-12 Years & $59(50.9 \%)$ & $57(49.1 \%)$ & \\
\hline
\end{tabular}

\section{DISCUSSION}

It has been an accepted phenomenon that patients suffering from psychiatric illness have low quality of life and they face difficulties in many domains of life but recently a lot of work has been done to look for the same phenomenon among the caregivers of psychiatric patients especially those suffering from chronic illnesses like psychotic illness. Caregivers of schizophrenic patients have been studied in this regard and found functioning less as compared to the normal population. ${ }^{7}$ Caregiving is not a single dimension phenomenon and it does not mean just looking after the patient for a specific time. An exhausting process involves many aspects which cover over all provision of care to a person who has been suffering from a long-standing illness. This predisposes the caregiver towards a lot of mental health problems. ${ }^{8}$

Data has been limited in our part of the world but few previous studies have shown similar findings as that of ours especially study done in Lahore has shown results which can be compared to the results of our study. ${ }^{9}$ In this study young age of caregivers and female gender had a strong link with presence of depression and anxiety among the caregivers of psychiatric patients. It is an interesting finding that most caregivers in our part of the world are females. They are generally more prone towards neurotic illness otherwise, the burden of caregiving adds to this tendency, and they fall prey to the psychiatric symptomatology themselves.

In present study, the average age observed was $35.36 \pm 8.39$ years. Frequency of Female caregiver is more as compared to male. A recent study form India found increased workload, related anxiety and depression, and sleep disturbance among 70\%, 76\%, and $43 \%$ of caregivers, respectively. Caregivers of the patients with dementia and depression experienced greater stress. Female caregivers were found to receive more appreciation and family bonding was well maintained. ${ }^{10}$ Carod-Artal et al found the prevalence of anxiety disorders was significantly higher in female caregivers than in males ( 26.1 vs. $10.9 \%$; $p=0.04$ ) as assessed by HADS. 11

In this study, frequency of depression among caregivers of psychiatric patients was found in $47.5 \%$. In a study by Alvi et al ${ }^{6}$ a significant number of caregivers was having depression and anxiety themselves. Similar results were reported in a study done in Turkey. ${ }^{12}$ Among all the psychiatric illness schizophrenia has been considered to be the most chronic illness in which most patients remain un well if not taking treatment and even with treatment 2/3rd of the patients remain symptomatic therefore care giving in these patients has been a real challenge.

Sometimes caregivers can under or over rate their burden therefore a comprehensive assessment especially of high risk caregivers is required in the clinical sessions. ${ }^{13}$ There are a lot of factors which have been associated with care giving burden. Family histories of psychiatric illness and number of patients in the family have also an implication on caregiving burden. Role of clinician has also been important in this regard. It has been observed that if proper information regarding the care has been provided by the treating physician to the caregiver it might reduce the burden. ${ }^{14}$ Goals of caregiving if achieved also alleviate the burden. It has been observed that if the condition of patient improves with the time and care giving of the caregiver it results in less burden and stress on the caregiver but if condi- 
tion of patient remains same or worsens then burden and perceived stress increase and depression and anxiety could be the final result. ${ }^{15}$

Other important factors which could be linked to the care giving burden and stress include stigma of mental health problems and the relationship of caregiver with the patient. Closer the relationship and emotional bond more the caregiver prone towards development of psychiatric disorders including depression and anxiety. ${ }^{16}$

No caregiver in our study was previously evaluated for any mental health issue. This was the first time they were asked about this phenomenon. Nobody in the past realized that they could face the stress and burden as well. Studies previously done in other parts of the world have concluded that these caregivers sometimes have been sole earners of their families and decline in their quality of life made the whole family suffer drastically. ${ }^{16-18}$

Spending more time with the patient and not receiving any rest or respite was significantly linked with depression and anxiety in the studies done in the past. ${ }^{19,20}$ Results of our study also reflected high burden of depression and anxiety among the people who were responsible for the care of those who were not well. Routine screening of caregivers and early detection and management of care giving burden or stress may improve the outcome of psychiatric problems among caregivers.

\section{CONCLUSION}

We found that depression was a highly common undiagnosed entity among the caregivers of psychiatric patients. Female caregivers or caregivers with low socio-economic status should be screened especially for depression in addition to caregivers of psychotic patients.

\section{Conflict of Interest: None.}

\section{Author Contribution}

NI: Conception, design, data collection, drafting. SH: Data analysis. SK: Data analysis. SK: Final drafting. MA: Conception \& design. MK: Conception \& design. SA: Final approval.

\section{REFERENCES}

1. Mustahsan SM, Shamim R, Mushtaq M, Farooque K, Razzaque $\mathrm{R}$, Hasnain SF, et al. The impact of hospital settings on the mood status of family caregivers. J Hosp Admin 2014; 3(5): 144-149.

2. FMH. Caregivers and mental illness living with shizophrenia. 2014 [Internet] Available at: http://www.fmh.com/wp-content/ uploads/2014/12/Caregivers-and-Mental-Illness.pdf (Accessed on December, 12 2017).
3. Minhas FA, Niazi RS. Depression and anxiety in the caregivers of mentally ill patients. J Pak Psych Soc 2005; 2(1): 27-33.

4. Rodrigo C, Fernando T, Raja-Pakse S, Silva VD, Habwella R. Caregivers strain and symptoms of depression among principal caregivers of patietns with schizophrenia and bipolar affective disorder in Sri Lanka. Int J Ment Health Sys 2013; 7(1): 2.

5. Imran N, Bhatti MR, Haider II, Azhar L, Omar A, Sattar A. Caring for the caregivers: Mental health, family burden and quality of life of caregivers of patietns with mental illnes. J Pak Psychiatric Soc 2010; 7(1): 23-26.

6. Alvi T, Hussain S, Assad F. Anxiety and depression among caregivers of Psychatric patietns in a tertiary care hospital from Pakistan. J Pak Psych Soc 2014; 11(1): 18-20.

7. Grover S, Chakrabarti S, Ghormode D, Dutt A, Kate N, Kulhara P. Clinicians' versus caregivers' ratings of burden in patients with schizophrenia and bipolar disorder. Int J Soc Psychiatr 2013; 60(4): 330-336.

8. Oshodi YO, Adeyemi JD, Aina OF, Suleiman TF, Erinfolami AR, Umeh C. Burden and psychological effects: caregiver experiences in a psychiatric outpatient unit in Lagos, Nigeria. Afr J Psychiatr 2012; 15(2): 99-105.

9. Imran N, Bhatti MR, Haider II, Azhar L, Omar A, Sattar A. Caring for the caregivers: mental health, family burden and quality of life of caregivers of patients with mental illness. J Pak Psychitr Assoc 2010; 7(1): 23-34.

10. Das S, Hazra A, Ray BK, Ghosal M, Banerjee TK, Roy T, et al. Burden among stroke caregivers: results of a community-based study from Kolkata, India. Stroke 2010; 41 (12): 2965-2968.

11. Carod-Artal FJ, Ferreira Coral L, Trizotto DS, Menezes Moreira C. Burden and perceived health status among caregivers of stroke patients. Cerebrovasc Dis 2009; 28 (5): 472-480.

12. Ak M, Yavuz KF, Lapsekili N, Türkçapar MH. Evaluation of burden in a group of patients with chronic psychiatric disorders and their caregivers. Düsünen Adam: J Psychiatry Neurological Sci 2012; 25(4): 330-337.

13. Perlick DA, Miklowitz DJ, Link BG, Struening E, Kaczynski R. Perceived stigma and depression among care-givers of patients with bipolar disorder. Br J Psychiatr 2007; 190: 535-536.

14. Jungbauer J, Bischkopf J, Angermeyer MC. Stress of family caregivers of psychiatric patients. Developmental trends, concepts and results of research. Psychiatrische Praxis 2001; 28(3): 105-114.

15. Schulz R, Sherwood PR. Physical and mental health effects of family caregiving. Am J Nursing 2008; 108(9 Suppl): 23-27.

16. Petrikis P, Baldouma A, Katsanos AH, Konitsiotis S, Giannopoulos S. Quality of life and emotional strain in caregivers of patients with multiple sclerosis. J Clin Neurol 2019; 15(1): 77-83.

17. O'Hara RE, Hull JG, Lyons KD, Bakitas M, Hegel MT, Li Z, Ahles TA. Impact on caregiver burden of a patient-focused palliative care intervention for patients with advanced cancer. Palliat Support Care 2010; 8(4): 395-404.

18. He J, Dai W, Li Y, He L, Huang R. Frequency of depression-related symptoms in caregivers of patients with silicosis. J Healthc Eng 2019; 2019: 6035920.

19. Del-Pino-Casado R, Espinosa-Medina A, López-Martínez C, Orgeta V. Sense of coherence, burden and mental health in caregiving: A systematic review and meta-analysis. J Affect Disord 2019; 242: 14-21.

20. Addo R, Agyemang SA, Tozan Y,. Economic burden of caregiving for persons with severe mental illness in sub-Saharan Africa: A systematic review. PLoS One 2018; 13(8): e0199830. 